\title{
TOPOLOGICAL TYPES OF KLEIN SURFACES WITH A MAXIMUM ORDER AUTOMORPHISM
}

\author{
by J. A. BUJALANCE $\dagger$
}

(Received 4 April, 1986)

1. Introduction. If $X$ is a Klein surface (KS) with boundary, of algebraic genus $p$, and $\Phi$ is an automorphism of order $N$, May [8] proved that $N \leq 2 p+2$ when $X$ is orientable and $p$ is even, and $N \leq 2 p$ otherwise.

He proved also that the unique topological type of an orientable KS having an orientation-preserving automorphism of maximum order is a surface with one boundary component when $p$ is even, with two boundary components when $p$ is odd.

In [8] May asks for the topological types of an orientable KS with boundary having an orientation-reversing automorphism of maximum order, and also for the topological types of non-orientable KS with boundary having an automorphism of maximum order.

In this work we solve this problem. We obtain besides, in all cases, the types of NEC groups providing the automorphism of maximum order. This result is also obtained for the case that May solved in [8].

We prove also that the maximum order of an orientation-reversing automorphism of an orientable KS with boundary, of genus $p$ odd, is $2 p-2$. This bound completes the list given by May [8] of the maximum order of automorphisms. The technique we use is the theory of NEC groups.

2. NEC groups and Klein surfaces. The first modern study of KS is due to Alling and Greenleaf [1]. They are surfaces with or without boundary endowed with a dianalytic structure; the automorphisms of these surfaces are dianalytic homeomorphisms.

In this work we study Klein surfaces using non-euclidean crystallographic groups (NEC groups) [6].

An NEC group is a discrete subgroup $\Gamma$ of the group of isometries of the non-euclidean plane $U(U=\{z \in \mathbb{C} / \operatorname{Im} z>0\})$ with compact quotient space, including orientation-reversing elements, reflections and glide-reflections. form

NEC groups are classified according to their signatures [6], which are symbols of the

$$
\left(g ; \pm ;\left[m_{1}, \ldots, m_{t}\right] ;\left\{\left(n_{11}, \ldots, n_{1 s_{1}}\right) \ldots\left(n_{k 1}, \ldots, n_{k s_{k}}\right)\right\}\right),
$$

where $g$ is the genus of the surface $U / \Gamma$, the sign \pm indicates whether the surface is orientable or not, the $m_{i} \geq 2$ (proper periods) represent the branching order of interior points of the surface by the canonical projection $p: U \rightarrow U / \Gamma$, the $n_{i j} \geq 2$ (periods of period-cycles) represent the branching order of boundary points of the surface by the above mapping, and $k$ is the number of holes of the surface.

† Partially supported by CAICYT.

Glasgow Math. J. 30 (1988) 87-96. 
The signature of an NEC group determines a presentation of it given by generators
(i) $x_{1}, \ldots, x_{t}$,
(ii) $e_{1}, \ldots, e_{k}$,
(iii) $c_{10}, \ldots, c_{1 s_{1}}, \ldots, c_{k 0}, \ldots, c_{k s_{k}}$,
(iv) (if the sign is + ) $a_{1}, b_{1}, \ldots, a_{g}, d_{g}$ (if the sign is - ) $d_{1}, \ldots, d_{g}$

and the relations

(i) $x_{i}^{m_{i}}=1, i=1, \ldots, t$,

(ii) $c_{i j-1}^{2}=c_{i j}^{2}=\left(c_{i j-1} c_{i j}\right)^{n_{i j}}=1\left(i=1, \ldots, k ; j=1, \ldots, s_{i}\right)$,

(iii) $e_{i}^{-1} c_{i 0} e_{i} c_{i_{i}}=1, i=1, \ldots, k$,

(iv) (if the sign is + )

$$
\begin{gathered}
x_{1} \ldots x_{t} e_{1} \ldots e_{k} a_{1} b_{1} a_{1}^{-1} b_{1}^{-1} \ldots a_{g} b_{a} a_{g}^{-1} b_{g}^{-1}=1 \\
\text { (if the sign is -) } x_{1} \ldots x_{t} e_{1} \ldots e_{k} d_{1}^{2} \ldots d_{g}^{2}=1 .
\end{gathered}
$$

From now on the letters $x, a, b, c, d, e$, will be used for these canonical generators of the group.

The relation between NEC groups and KS is a consequence of the following results of Preston [9], Alling-Greenleaf [1] and May [7].

If $X$ is a KS of algebraic genus $p \geq 2$, then $X$ may be represented by $U / \Gamma$, where $\Gamma$ is an NEC group with signature

$$
(g ; \pm ;[-] ;\{\underbrace{(-) \ldots(-)}_{k}\}) .
$$
of $\mathrm{KS}$.

If $\Gamma$ is an NEC group then the quotient space $U / \Gamma$ may be endowed with a structure

A group $H$ is an automorphism group of the KS $U / \Gamma$ if and only if $H \cong \Gamma^{\prime} / \Gamma$ where $\Gamma^{\prime}$ is an NEC group such that $\Gamma \triangleleft \Gamma^{\prime}$ ( $\Gamma$ a bordered surface group).

An NEC group with orientation-reversing elements will be called a proper NEC group.

If $\Gamma$ is a proper NEC group, then the Fuchsian subgroup of $\Gamma$, formed by its orientation-preserving elements, has index 2 in $\Gamma$ and will be denoted by $\Gamma^{+}$(canonical Fuchsian group of $\Gamma$ ).

We shall indicate now some results obtained in $[2,3]$ that will be used throughout this paper.

THEOREM 2.1 [2]. Let $\Gamma$ be an NEC group with signature

$$
\left(g ;+;\left[m_{1}, \ldots, m_{t}\right] ;\left\{\left(n_{11}, \ldots, n_{1 s_{1}}\right) \ldots\left(n_{k 1}, \ldots, n_{k s_{k}}\right)\right\}\right)
$$


and $\Gamma_{0}$ a normal subgroup of $\Gamma$ such that $\left[\Gamma: \Gamma_{0}\right]=N, N$ even. We suppose that:

(i) $C=\left\{c_{j}, c_{j}^{\prime}\right\}(j=1, \ldots, p)$ is the set of pairs of reflections which are generators of $\Gamma$, not belonging to $\Gamma_{0}$, and such that $c_{j} . c_{j}^{\prime}$ is an elliptic element of order $n_{j}$;

(ii) $p_{i}$ is the exponent of $x_{i}$ modulo $\Gamma_{0}(1 \leq i \leq t)$;

(iii) $q_{j}$ is the exponent of $c_{j} . c_{j}^{\prime}$ modulo $\Gamma_{0}(1 \leq j \leq p)$.

Then the proper periods of $\Gamma_{0}$ are

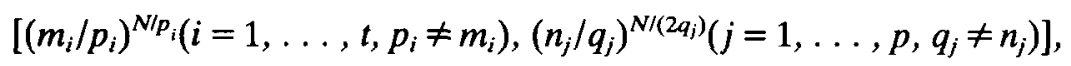

where by $(-)^{r}$ we mean that this proper period is repeated $r$ times.

THEOREM 2.2 [3]. Let $\Gamma$ be an NEC group with signature

$$
\sigma(\Gamma)=(g ; \pm ;[--] ;\{\underbrace{(-) \ldots(-)}_{k}\}),
$$

and $\Gamma^{\prime}$ an NEC group such that $\Gamma \triangleleft \Gamma^{\prime}$ and $\Gamma^{\prime} / \Gamma \cong Z_{N}, N$ even. Then $\Gamma^{\prime}$ has signature

$$
\sigma\left(\Gamma^{\prime}\right)=\left(g ; \pm ;\left[N / k_{1}, \ldots, N / k_{t}\right] ;\{\underbrace{(-) \ldots(-)}_{s}(\underbrace{2, \ldots, 2}_{r_{i}^{\prime}})^{r_{1}} \cdots(\underbrace{2, \ldots, 2}_{r_{k^{\prime}}})^{r_{k^{\prime}}}\}\right),
$$

where the numbers $r_{i}$ are even and

$$
k=s-p-t^{\prime}+\sum_{\substack{n \in N \\ n \neq N}} t_{n} N \mid n+\sum_{j=1}^{k^{\prime}} r_{j} N / 4,
$$

with $t^{\prime}=\sum_{\substack{n \mid N \\ n \neq N}} t_{n}$ and $t_{n} \geq 0$, where $t_{n}$ and $p$ are positive integers.

3. Topological types. If $X=U / \Gamma$ is a compact orientable KS of algebraic genus at least two and $\Phi$ is an automorphism of $X$, it is known that $\langle\Phi\rangle \cong \Gamma^{\prime} / \Gamma$ where $\Gamma^{\prime}$ is an NEC group and $\langle\Phi\rangle$ denotes the group generated by $\Phi$. Besides if $g \Gamma$ is a generator of $\Gamma^{\prime} / \Gamma$, one may define

3. Topological types. If $X=U / \Gamma$ is a compact orientable KS of algebraic genus at least two and $\Phi$ is an automorphism of $X$, it is known that $\langle\Phi\rangle \cong \Gamma^{\prime} / \Gamma$ where $\Gamma^{\prime}$ is an

TheOREM 3.1. Let $X=U / \Gamma$ be an orientable bordered Klein surface and $G \cong \Gamma^{\prime} / \Gamma$ the automorphism group of $X$. Then all the elements of $G$ preserve the orientation of $X$ if and only if all orientation-reversing elements of the canonical generating system of $\Gamma^{\prime}$ belong to $\Gamma$.

Proof. Let $R^{\prime}$ be a fundamental region of $\Gamma^{\prime}$ (see [11]) and $N$ the order of $G$. Then if

$$
\Gamma^{\prime} / \Gamma \cong\left\{y_{1} \Gamma, y_{2} \Gamma, \ldots, y_{N-1} \Gamma, \Gamma\right\}
$$

we have that

$$
R=R^{\prime} \cup y_{1}\left(R^{\prime}\right) \cup \ldots \cup y_{N-1}\left(R^{\prime}\right)
$$


is a fundamental region of $\Gamma$; moreover, applying $\Gamma$ to $R$, we obtain a tessellation of $U$, and $R / \Gamma \cong U / \Gamma$.

Suppose that not all reflections or glide reflections of the canonical generator system of $\Gamma^{\prime}$ belong to $\Gamma$; then let $c$ be a reflection of $\Gamma^{\prime}$ not belonging to $\Gamma$ : we may take $y_{i}=c$; if we write $R^{\prime}$ as $A \tau$ where $\tau$ is the side fixed by $c$ and $A$ denotes the other sides of the polygon, we have in $R$ the connected region $R^{\prime} \cup c\left(R^{\prime}\right)$. Suppose that $c \Gamma$ is the element of $\Gamma^{\prime} / \Gamma$ corresponding to an automorphism $\Phi \in G$; then $\Phi$ reverses the orientation of the interior of $R$ since the image of $R^{\prime}$ associated to this automorphism is $c\left(R^{\prime}\right)$. Hence $\Phi$ reverses the orientation of $U / \Gamma$.

We would act the same way if there were a glide reflection of $\Gamma^{\prime}$ not belonging to $\Gamma$.

We now prove the sufficiency. Let all orientation-reversing elements of the canonical generating system of $\Gamma^{\prime}$ belong to $\Gamma$ and suppose that $g \Gamma \in \Gamma^{\prime} / \Gamma$.

Let $g=w_{1} \ldots w_{k-1} w_{k} w_{k+1} \ldots w_{r}$ be an expression for $g$ in terms of the canonical generators of $\Gamma^{\prime}$, where $w_{k}$ reverses orientation. Then

$$
\begin{aligned}
g \Gamma & =\left(w_{1} \ldots w_{k-1}\right) w_{k}\left(w_{k+1} \ldots w_{r}\right) \Gamma=\left(w_{1} \ldots w_{k-1}\right) w_{k} \Gamma w_{k+1} \ldots w_{r} \\
& =w_{1} \ldots w_{k-1} w_{k+1} \ldots w_{r} \Gamma,
\end{aligned}
$$

as $w_{k} \in \Gamma$. Thus we can choose all the coset representatives to be orientation-preserving.

Given $\Phi \in G$, let $y_{i} \Gamma$ be the element of $\Gamma^{\prime} / \Gamma$ associated to $\Phi$. Define now an orientation in the interior of $y_{s} R^{\prime}$; since $y_{i}$ preserves the orientation, $y_{i} y_{s} R^{\prime}$ has the same orientation. The identification of sides of $y_{s} R^{\prime}$ and $y_{i} y_{s} R^{\prime}$ is made by elements of $\Gamma^{\prime}$ preserving the orientation (non-orientable generators of $\Gamma^{\prime}$ belong to $\Gamma$ ), so $\Phi$ preserves the orientation of $U / \Gamma$.

THEOREM 3.2. Let $X=U / \Gamma$ be an orientable $K S$ with $k$ boundary components of algebraic genus $p \geq 2$.

(a) If $p$ is even and $X$ has an orientation-reversing automorphism of order $2 p+2$, then $k=p+1$. Besides, if $\Phi$ is an (orientation-reversing) automorphism of $X$ of order $2 p+2$, the group generated by it is $\langle\Phi\rangle \cong \Gamma^{\prime} / \Gamma$ where $\Gamma^{\prime}$ is an NEC group with signature

$$
(0 ;+;[p+1] ;\{(2,2)\}) \text {. }
$$

(b) If $p$ is odd, the maximum order of an orientation-reversing automorphism of $X$ is $2 p-2$, and this value is only obtained when $k=2$ and $4 \nmid p-3$ or when $k=4$ and $4 \mid p-3$. Besides if $\Phi$ is an orientation-reversing automorphism of $X$ of order $2 p-2$, the group generated by it is $\langle\Phi\rangle \cong \Gamma^{\prime} / \Gamma$, where $\Gamma^{\prime}$ is an NEC group with signature

$$
(1 ;-;[2] ;\{(-)\}) \text {. }
$$

Proof. (a) If $p$ is even, the maximum order of an orientation-reversing automorphism is $2 p+2$. If this value is achieved, there exists an NEC group $\Gamma^{\prime}$ with $\Gamma^{\prime} / \Gamma \cong Z_{2 p+2}$.

From the relation of areas of the fundamental regions (see [11]) of $\Gamma$ and $\Gamma^{\prime}$ one has $\left|\Gamma^{\prime}\right|=|\Gamma| /(2 p+2)$. Since the area of $\Gamma$ (see [10]) (up to a factor $2 \pi$ ) is $p-1$, one has

$$
\left|\Gamma^{\prime}\right|=1 / 2-1 /(p+1) \text {. }
$$


Since $\Gamma$ has period-cycles, $\Gamma^{\prime}$ also has period-cycles.

If $\Gamma^{\prime}$ has sign + in its signature, by 2.2 the signature of $\Gamma^{\prime}$ has the form

$$
\left(g^{\prime} ;+;\left[(2 p+2) / k_{1}, \ldots,(2 p+2) / k_{t}\right] ;\{\underbrace{(-) \ldots(-)}_{s}(\underbrace{2, \ldots, 2}_{r_{i}^{\prime}})^{r_{1}} \cdots(\underbrace{2, \ldots, 2}_{r_{k^{\prime}}})^{r_{k^{\prime}}}\}\right),
$$

and so its area is, up to a factor $2 \pi$,

$$
\left|\Gamma^{\prime}\right|=k_{0}+2 g^{\prime}-2+1 / 2 \sum_{i=1}^{k^{\prime}} \sum_{j=1}^{r_{i} r_{i}^{\prime}}(1-1 / 2)+\sum_{i=1}^{t}\left(1-k_{i} /(2 p+2)\right),
$$

where $k_{0}$ is the number of period-cycles in the signature of $\Gamma^{\prime}$, and so $k_{0} \geq 1$.

By (1), $\left|\Gamma^{\prime}\right|<1 / 2$ and as $k_{0} \geq 1$ we have $g^{\prime}=0$. We then see that $k_{0}=1$ and now from (1) and (2)

(i) $k^{\prime}=1, r_{1}^{\prime} r_{1}=0, t=2$

or

(ii) $k^{\prime}=1, r_{1}^{\prime} r_{1}=2, t=1$.

In case (i) we have from (1) and (2)

$$
1 / 2-1 /(p+1)=-1+\sum_{i=1}^{2}\left(1-k_{i} / 2(p+1)\right)
$$

and hence $k_{1}+k_{2}-3=p$. As $k_{1}$ and $k_{2}$ both divide $2(p+1)$ the only solution is $k_{1}=p+1, k_{2}=2$.

Analogously in case (ii) one obtains $k_{1}=2$.

Hence the signatures of $\Gamma^{\prime}$ are

$$
\text { (a) }(0 ;+;[2, p+1] ;\{(-)\})
$$

or

$$
\text { ( } \beta)(0 ;+;[p+1] ;\{(2,2)\}) \text {. }
$$

If the signature of $\Gamma^{\prime}$ is $(\alpha)$ then there is just one conjugacy class of reflections in $\Gamma^{\prime}$. As $U / \Gamma$ has a boundary, $\Gamma$ also has reflections. Thus if $c \in \Gamma^{\prime} / \Gamma$ is a reflection and $\theta: \Gamma^{\prime} \rightarrow Z_{2 p+2}$ is the homomorphism then $\theta(c)=\overline{0}$ so by Theorem 3.1 the automorphism associated to $\theta$ is orientable. In case $(\beta)$ there exists an epimorphism

$$
\theta: \Gamma^{\prime} \rightarrow Z_{2 p+2}
$$

with $\operatorname{ker} \theta \cong \Gamma$, such that

$$
\left\{\begin{array}{l}
\theta\left(c_{1}\right)=\overline{p+1} \\
\theta\left(c_{2}\right)=\overline{0}, \\
\theta\left(c_{3}\right)=\overline{p+1}
\end{array}\right\} \quad \text { or } \quad\left\{\begin{array}{l}
\theta\left(c_{1}\right)=\overline{0}, \\
\theta\left(c_{2}\right)=\overline{p+1} \\
\theta\left(c_{3}\right)=\overline{0} .
\end{array}\right\}
$$

By $2.1 \theta\left(x_{1}\right)=\bar{a}$ has order $p+1$, and so $\theta\left(e_{1}\right)=\overline{2 p+2-a}$, since $\theta\left(x_{1} e_{1}\right)$ is $\overline{0}$; hence by 
Theorem 3.1 the automorphism associated to $\theta$ reverses the orientation. As $c_{1} c_{3} \in \operatorname{ker} \theta$, by $[4,3.1] k=(2 p+2) / 2=p+1$.

If $\Gamma^{\prime}$ has sign - in its signature, then by 2.2 it has the form

$$
\left(g^{\prime} ;-;\left[(2 p+2) / k_{1}, \ldots,(2 p+2) / k_{t}\right] ;\{\underbrace{(-) \ldots(-)}_{s}(\underbrace{2, \ldots, 2}_{r_{i}^{\prime}})^{r_{1}} \cdots(\underbrace{2, \ldots, 2}_{r_{k^{\prime}}})^{r_{k}}\}\right),
$$

and so the area of the associated fundamental region (up to factor $2 \pi$ ) is

$$
\left|\Gamma^{\prime}\right|=k_{0}+g^{\prime}-2+1 / 2 \sum_{i=1}^{k^{\prime}} \sum_{j=1}^{r_{i} r_{i}^{\prime}}(1-1 / 2)+\sum_{i=1}^{t}\left(1-k_{i} /(2 p+2)\right),
$$

where $k_{0}$ is the number of period-cycles of the signature of $\Gamma^{\prime}$ and so $k_{0} \geq 1$. As $g^{\prime} \geq 1$, then $\left|\Gamma^{\prime}\right| \geq 1 / 2$ (since $r^{\prime}$ is even), and so (3) is never equal to (1).

(b) If $p$ is odd, then by [8] the maximum possible order of an orientation-reversing automorphism of $X$ is $2 p$. If this bound is attained, there exists an NEC group $\Gamma^{\prime}$ with $\Gamma^{\prime} / \Gamma \cong Z_{2 p}$.

From the relation of the areas of fundamental regions of $\Gamma$ and $\Gamma^{\prime}$ one has that $\left|\Gamma^{\prime}\right|=|\Gamma| /(2 p)$.

Since the area of $\Gamma$ (up to a factor $2 \pi$ ) is $p-1$, we have

$$
\left|\Gamma^{\prime}\right|=1 / 2-1 / 2 p \text {. }
$$

If the signature of $\Gamma^{\prime}$ has sign + , then by 2.2 it has the form

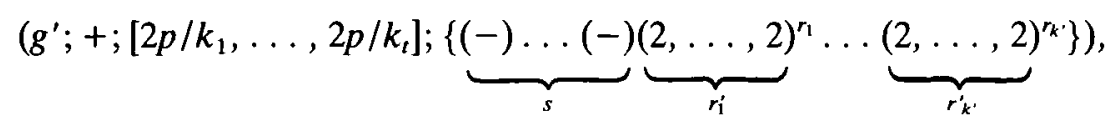

and so its area is

$$
\left|\Gamma^{\prime}\right|=k_{0}+2 g^{\prime}-2+1 / 2 \sum_{i=1}^{k^{\prime}} \sum_{j=1}^{r_{r}^{\prime} r_{i}^{\prime}}(1-1 / 2)+\sum_{i=1}^{t}\left(1-k_{i} / 2 p\right)
$$

where $k_{0} \geq 1$.

From (4) and (5) we have $g^{\prime}=0$; besides, $k_{0}$ cannot be 2 since then the remaining quantities of (5) would have a value bigger than $1 / 2$; if $k_{0}=1$ the only solutions for coincidence of values of (5) and (4) are:

$$
\begin{aligned}
& (0 ;+;[2 p] ;\{(2,2)\}), \\
& (0 ;+;[2,2 p] ;\{(-)\}), \\
& (0 ;+;[3,3] ;\{(-)\}), p=3, \\
& (0 ;+;[3,5] ;\{(-)\}), p=15,
\end{aligned}
$$

for which there does not exist the epimorphism we are looking for.

Since there are not automorphisms with maximum order $2 p$, we look for automorphisms of order $2 p-2$. 
The relation of areas is now

$$
\left|\Gamma^{\prime}\right|=|\Gamma| /(2 p+2)=(p-1) /(2 p-2)=1 / 2 .
$$

If $\Gamma^{\prime}$ has sign + in its signature, then by 2.2 that signature has the form

$$
\left(g^{\prime} ;+;\left[(2 p-2) / k_{1}, \ldots,(2 p-2) / k_{t}\right] ;\{\underbrace{-(-) \ldots(-)}_{s}(\underbrace{2, \ldots, 2)^{r_{1}}}_{r_{\mathrm{i}}} \cdots(\underbrace{(2, \ldots, 2}_{r_{k^{\prime}}})^{r_{k^{\prime}}}\})\right.
$$

and so the area is (up to a factor $2 \pi$ )

$$
\left|\Gamma^{\prime}\right|=k_{0}+2 g^{\prime}-2+1 / 2 \sum_{i=1}^{k^{\prime}} \sum_{j=1}^{r_{i} r_{i}^{\prime}}(1-1 / 2)+\sum_{i=1}^{t}\left(1-k_{i} /(2 p-2)\right),
$$

where $k_{0} \geq 1$. Then the only solutions equating (6) and (7) are

$$
\begin{aligned}
& \text { (i) }(0 ;+;[-] ;\{(2,2,2,2,2,2)\}) \text {, } \\
& \text { (ii) }(0 ;+;[2] ;\{(2,2,2,2)\}) \text {, } \\
& \text { (iii) }(0 ;+;[2,2] ;\{(2,2)\}) \text {, } \\
& \text { (iv) }(0 ;+;[2,2,2] ;\{(-)\}) \text {, } \\
& \text { (v) }(0 ;+;[4,4] ;\{(-)\}) \text {, } \\
& \text { (vi) }(0 ;+;[3,6] ;\{(-)\}) \text {, } \\
& \text { (vii) }(0 ;+;[2] ;\{(-)(-)\}) \text {, } \\
& \text { (viii) }(0 ;+;[-] ;\{(2,2)(-)\}) \text {. }
\end{aligned}
$$

In cases (i), (ii), (iii), (iv), if there exists an epimorphism $\theta: \Gamma^{\prime} \rightarrow Z_{2 p-2}$ with any of these signatures, then by $[4,3.1]$ and 2.1 the group $Z_{2 p-2}$ would have only generators of order 2 , whence $p=2$, which is impossible.

In case (v) there exists no epimorphism whose associated automorphism is non-orientable.

In case (vii) if there exists $\theta: \Gamma^{\prime} \rightarrow Z_{2 p-2}$ with $\operatorname{ker} \theta \cong \Gamma$, then by $[3,3.1]$ and 2.1 one has

$$
\begin{aligned}
& \theta\left(x_{1}\right)=\overline{p-1} \text { of order } 2, \\
& \theta\left(c_{1}\right)=\overline{0} \text { or } p-1, \\
& \theta\left(c_{2}\right)=\overline{0} \text { or } p-1, \\
& \theta\left(e_{1}\right)=\bar{b} \\
& \theta\left(e_{2}\right)=\theta\left(e_{1}^{-1} x_{1}^{-1}\right) .
\end{aligned}
$$

Since the automorphism associated to $\theta$ is non-orientable, then by Theorem 3.1 $\theta\left(c_{1}\right)=\overline{p-1}$ or $\theta\left(c_{2}\right)=\overline{p-1}$, whence $\theta\left(x_{1} c_{1}\right)=\overline{0}$ or $\theta\left(x_{1} c_{2}\right)=\overline{0}$, and hence (see [5, Theorem 2]) $\Gamma$ would have sign - in its signature, which is impossible. 
In case (viii), by $[3,3.1]$ one has

$$
\begin{aligned}
& \left\{\begin{array}{l}
\theta\left(c_{10}\right)=\overline{p-1}, \\
\theta\left(c_{11}\right)=\overline{0}, \\
\theta\left(c_{12}\right)=\overline{p-1}
\end{array}\right\} \text { or }\left\{\begin{array}{l}
\theta\left(c_{10}\right)=\overline{0}, \\
\theta\left(c_{11}\right)=\overline{p-1} \\
\theta\left(c_{12}\right)=\overline{0},
\end{array}\right\} \\
& \theta\left(c_{20}\right)=\overline{0} \text { or } \overline{p-1} .
\end{aligned}
$$

Since $\theta\left(e_{2}\right)=\theta\left(e_{1}^{-1}\right)$, one has $\theta\left(e_{1}\right)$ and $p-1$ generate $Z_{2 p-2}$; as $2(p-1)$ is a multiple of $4, \theta\left(e_{1}\right)$ must have order $2(p-1)$, since otherwise the two elements would not generate the group. Supposing $\theta\left(e_{1}\right)=\overline{1}$ (in other cases we act analogously) one obtains $\theta\left(e_{1}^{p-1} c_{10}\right)=\overline{0}$, and so ker $\theta$ has sign - in its signature (which is not possible).

If $\Gamma^{\prime}$ has sign - in its signature, then by 2.2 the signature of $\Gamma^{\prime}$ has the form

$$
\left(g ;-;\left[(2 p-2) / k_{1}, \ldots,(2 p-2) / k_{t}\right] ;\{\underbrace{(-) \ldots(-)}_{s}(\underbrace{2, \ldots, 2}_{r_{1}^{\prime}})^{r_{1}} \cdots(\underbrace{2, \ldots, 2}_{r^{\prime} k^{\prime}})^{r^{*}}\}\right),
$$

and so the area is (up to a factor $2 \pi$ )

$$
\left|\Gamma^{\prime}\right|=k_{0}+g^{\prime}-2+1 / 2 \sum_{i=1}^{k^{\prime}} \sum_{i=1}^{r_{i} r_{i}^{\prime}}(1-1 / 2)+\sum_{i=1}^{t}\left(1-k_{i} /(2 p-2)\right),
$$

where $k_{0} \geq 1$ and $g^{\prime} \geq 1$. The only solutions for (6) equating with (8) are:

(i) $(1 ;-;[-] ;\{(2,2)\})$,

(ii) $(1 ;-;[2] ;\{(-)\})$.

If $\Gamma^{\prime}$ has signature (i) and there exists an epimorphism $\theta: \Gamma^{\prime} \rightarrow Z_{2 p-2}$ with $\operatorname{ker} \theta \cong \Gamma$, we have, by $[3,3.1]$

$$
\left\{\begin{array}{l}
\theta\left(c_{10}\right)=\overline{p-1} \\
\theta\left(c_{11}\right)=\overline{0}, \\
\theta\left(c_{12}\right)=\overline{p-1}
\end{array}\right\} \text { or }\left\{\begin{array}{l}
\theta\left(c_{10}\right)=\overline{0}, \\
\theta\left(c_{11}\right)=\overline{p-1} \\
\theta\left(c_{12}\right)=\overline{0}
\end{array}\right\}
$$

with $\theta\left(e_{1}\right)=\theta\left(d_{1}^{-2}\right)$; so $p-1$ and $\theta\left(d_{1}\right)$ generate $Z_{2 p-2}$. An argument similar to the case (viii) above allows us, without loss of generality, to suppose that $\theta\left(d_{1}\right)=\overline{1}$; from which one deduces $\theta\left(d_{1}^{p-1} c_{10}\right)=\overline{0}$ and $d_{1}^{p-1} c_{10}$ is a non-orientable element since $p-1$ is even; hence $\operatorname{ker} \theta$ would have sign - in its signature and it would not be isomorphis to $\Gamma$.

If $\Gamma^{\prime}$ has signature (ii), any epimorphism $\theta: \Gamma^{\prime} \rightarrow Z_{2 p-2}$ with $\operatorname{ker} \theta \cong \Gamma$ should satisfy

$$
\begin{aligned}
& \theta\left(x_{1}\right)=\overline{p-1}, \\
& \theta\left(c_{10}\right)=\overline{0}, \\
& \theta\left(e_{1}\right)=\theta\left(x_{1}^{-1} d_{1}^{-2}\right) .
\end{aligned}
$$

As $\overline{p-1}$ and $\theta\left(d_{1}\right)$ generate $Z_{2 p-2}$ we may suppose $\theta\left(d_{1}\right)=\overline{1}$, and so $\theta\left(e_{1}\right)=\overline{p-3}$. By [5, Theorem 2] $U / \operatorname{ker} \theta$ is an orientable $K S$, and the automorphism associated to $\theta$ is non-orientable. 
Now we look for the number of boundary components of $U / \operatorname{ker} \theta$; by [4, Lemma 1] it is enough to know the minimum power of $e_{1}$ belonging to $\operatorname{ker} \theta$. If it is $\alpha$, then $\alpha(p-3)=n(2 p-2), n$ being an integer number, and $\alpha$ divides $p-1$, since $p-3$ is even. Hence $\alpha=2 n(p-1) /(p-3)=2 n+2(2 n /(p-3))$. As $\alpha \leq p-1$, we have

$$
2 n(p-1) /(p-3) \leq p-1,
$$

and so $2 n /(p-3) \leq 1$. As $\alpha$ is an integer, we have

$$
2 n /(p-3)=1 \text { or } 2 n /(p-3)=1 / 2 \text {, }
$$

and so $p=3+2 n$ or $p=3+4 n$.

If $p=3+2 n$, we have $\alpha=p-1$. If $p=3+4 n$ we have $\alpha=(p-1) / 2$. So, if $p-3=\dot{4}$, the minimum power $\alpha$ of $e_{1}$ such that $\theta\left(e_{1}^{\alpha}\right)=\overline{0}$ is $(p-1) / 2$. Hence by $[4$, Lemma 1], $k=2(p-1) /(p-1)=2$.

If $p-3 \neq \dot{4}$, the minimum power $\alpha$ of $e_{1}$ with $\left(e_{1}^{\alpha}\right)=\overline{0}$ is $p-1$, and by [4, Lemma 1] we have $k=2(p-1) /(p-1)=2$.

In a similar way we have the following theorems; part of the next theorem appears in May [8].

THEOREM 3.3. Let $X=U / \Gamma$ be an orientable $K S$ with $k$ boundary components of algebraic genus $p \geq 2$.

(a) If $p$ is even and $X$ has an orientation-preserving automorphism of order $2 p+2$, then $k=1$. Besides, if $\Phi$ is an automorphism of $X$ of order $2 p+2$, the group generated by it is $\langle\Phi\rangle \cong \Gamma^{\prime} / \Gamma$, where $\Gamma^{\prime}$ is an NEC group with signature

$$
(0 ;+;[2, p+1] ;\{(-)\}) .
$$

(b) If $p$ is odd and $X$ has an orientation-preserving automorphism of order $2 p$, then $k=2$. Besides, if $\Phi$ is an automorphism of $X$ of order $2 p$, the group generated by it is $\langle\Phi\rangle \cong \Gamma^{\prime} / \Gamma$, and $\Gamma^{\prime}$ is an NEC group with signature

$$
(0 ;+;[2,2 p] ;\{(-)\}) \text {. }
$$

THEOREM 3.4. Let $X=U / \Gamma$ be a non-orientable $K S$ with $k$ boundary components, of algebraic genus $p \geq 2$.

If $X$ has an automorphism of order $2 p$, then $k=p$. Besides, if $\Phi$ is an automorphism of $X$ of order $2 p$, the group generated by it is $\langle\Phi\rangle \cong \Gamma^{\prime} / \Gamma$, and $\Gamma^{\prime}$ is an NEC group with signature

$$
(0 ;+;[2 p] ;\{(2,2)\}) \text {. }
$$

This paper forms part of the doctoral thesis of the author, written under the direction of Prof. E. Bujalance. I express to him my acknowledgement.

The author wishes to thank the referee for his helpful suggestions.

\section{REFERENCES}

1. N. L. Alling and N. Greenleaf, Foundations of the theory of Klein surfaces, Lecture Notes in Mathematics 219 (Springer, 1971). 
2. E. Bujalance, Proper periods of normal NEC subgroups with even index, Rev. Mat. Hisp.-Amer. (4) 41 (1981), 121-127.

3. J. A. Bujalance, Normal subgroups of even index of an NEC group, Arch. Math. (Basel), to appear.

4. J. J. Etayo, On the order of automorphism groups of Klein surfaces, Glasgow Math. J. 26 (1985), 75-81.

5. A. H. M. Hoare and D. Singerman, The orientability of subgroups of plane groups, in C. M. Campbell and E. F. Robertson, eds., Groups-St. Andrews 1981, London Mathematical Society Lecture Note Series 71 (Cambridge, 1982), 221-227.

6. A. M. Macbeath, The classification of non-euclidean plane crystallographic groups, Canad. J. Math. 6 (1967), 1192-1205.

7. C. L. May, Large automorphism groups of compact Klein surfaces with boundary, Glasgow Math. J. 18 (1977), 1-10.

8. C. L. May, Cyclic automorphism groups of compact bordered Klein surfaces, Houston J. Math. 3 (1977), 395-405.

9. R. Preston, Projective structures and fundamental domains on compact Klein surfaces (University of Texas Ph.D. thesis, 1975).

10. D. Singerman, On the structure of non-euclidean crystallographic groups, Proc. Cambridge Philos. Soc. 76 (1974), 233-240.

11. M. C. Wilkie, On non-euclidean crystallographic groups, Math. Z. 91 (1966), 87-102.

\section{J. A. Bujalance}

Dep. De Matematicas

Centro Universitario de Toledo

TOLEDO

SPAIN 